\title{
Article \\ An Application of Sălăgean Operator Concerning Starlike Functions ${ }^{\dagger}$
}

\author{
Hatun Özlem Güney ${ }^{1}\left(\mathbb{D}\right.$, Georgia Irina Oros ${ }^{2}\left(\mathbb{D}\right.$ and Shigeyoshi Owa ${ }^{3, *} \mathbb{(}$ \\ 1 Department of Mathematics, Faculty of Science Dicle University, Diyarbakır 21280, Turkey; \\ ozlemg@dicle.edu.tr \\ 2 Department of Mathematics and Computer Sciences, Faculty of Informatics and Sciences, \\ University of Oradea, 410087 Oradea, Romania; georgia_oros_ro@yahoo.co.uk \\ 3 "1 Decembrie 1918" University of Alba Iulia, 510009 Alba Iulia, Romania \\ * Correspondence: shige21@ican.zaq.ne.jp \\ + Dedicated to the memory of Professor Yaşar Polatoğlu.
}

check for

updates

Citation: Güney, H.Ö.; Oros, G.I.;

Owa, S. An Application of Sălăgean Operator Concerning Starlike

Functions. Axioms 2022, 11, 50. https://doi.org/10.3390/ axioms11020050

Academic Editor: Kurt Bernardo Wolf

Received: 15 December 2021 Accepted: 25 January 2022 Published: 27 January 2022

Publisher's Note: MDPI stays neutral with regard to jurisdictional claims in published maps and institutional affiliations.

Copyright: (C) 2022 by the authors Licensee MDPI, Basel, Switzerland. This article is an open access article distributed under the terms and conditions of the Creative Commons Attribution (CC BY) license (https:// creativecommons.org/licenses/by/ $4.0 /)$
Abstract: As an application of the well-known Sălăgean differential operator, a new operator is introduced and, using this, a new class of functions $S_{n}(\alpha)$ is defined, which has the classes of starlike and convex functions of order $\alpha$ as special cases. Original results related to the newly defined class are obtained using the renowned Jack-Miller-Mocanu lemma. A relevant example is given regarding the applications of a new proven result concerning interesting properties of class $S_{n}(\alpha)$.

Keywords: analytic function; starlike function of order $\alpha$; convex function of order $\alpha$; Sălăgean differential operator; Alexander integral operator

\section{Introduction and Preliminaries}

Many operators have been used since the beginning of the study of analytic functions. The most interesting of these are the differential and integral operators. Since the beginning of the 20th century, many mathematicians, especially J.W. Alexander [1], S.D. Bernardi [2] and R.J. Libera [3], have worked on integral operators. It has become easier to introduce new classes of univalent functions with the use of operators. In his article, published in 1983, Sălăgean introduced differential and integral operators, which bear his name. Those operators were very inspiring and many mathematicians have obtained new, interesting results using these operators. In particular, researchers have introduced many new operators, examined their properties, and further used the newly defined operators to introduce classes of univalent functions with remarkable properties. At the same time, some mathematicians obtained interesting results in different lines of research by combining differential and integral operators, where Sălăgean differential operator was involved, as is seen, for example, in very recent papers [4-6]. The topic of strong differential subordination was also approached recently using Sălăgean differential operator in [7], and new operators were introduced using a fractional integral of Sălăgean and Ruscheweyh operators in [8]. The operators introduced using the Sălăgean differential operator were also recently used to obtain results related to the celebrated Fekete-Szegö inequality [9].

In this work, we introduce a new class as an application of the Sălăgean operator and discuss some interesting problems with this class.

Let $A$ be the class of functions $f$ of the form

$$
f(z)=z+\sum_{k=2}^{\infty} a_{k} z^{k}
$$


which are analytic in the open unit disc $\mathbb{U}=\{z \in \mathbb{C}:|z|<1\}$ and $\mathcal{S}$ be the subclass of $A$ consisting of univalent functions. Also,

$$
S^{*}(\alpha)=\left\{f \in A: \operatorname{Re}\left(\frac{z f^{\prime}(z)}{f(z)}\right)>\alpha, z \in \mathbb{U}, 0 \leq \alpha<1\right\}
$$

is the class of starlike functions of order $\alpha$ and

$$
K(\alpha)=\left\{f \in A: \operatorname{Re}\left(1+\frac{z f^{\prime \prime}(z)}{f^{\prime}(z)}\right)>\alpha, z \in \mathbb{U}, 0 \leq \alpha<1\right\}
$$

is the class of convex functions of order $\alpha$.

Let us start by recalling the well-known definitions for the Sălăgean differential and integral operators.

Definition 1 (Sălăgean [10]). For $f \in A$, the Sălăgean differential operator $D^{n}$ is defined by $D^{n}: A \rightarrow A$,

$$
\begin{gathered}
D^{0} f(z)=f(z)=z+\sum_{k=2}^{\infty} a_{k} z^{k}, \\
D^{1} f(z)=D f(z)=z f^{\prime}(z)=z+\sum_{k=2}^{\infty} k a_{k} z^{k}, \\
D^{n} f(z)=D\left(D^{n-1} f(z)\right)=z+\sum_{k=2}^{\infty} k^{n} a_{k} z^{k} \quad(n=1,2,3, \cdots),
\end{gathered}
$$

and Sălăgean integral operator $D^{-n}$ is defined by

$$
D^{-1} f(z)=\int_{0}^{z} \frac{f(t)}{t} d t=z+\sum_{k=2}^{\infty} \frac{1}{k} a_{k} z^{k}
$$

and

$$
D^{-n} f(z)=D^{-1}\left(D^{-n+1} f(z)\right)=z+\sum_{k=2}^{\infty} \frac{1}{k^{n}} a_{k} z^{k} \quad(n=1,2,3, \cdots) .
$$

In view of Definition 1, the following new operator is introduced:

Definition 2. For $f \in A$

$$
D^{j} f(z)=z+\sum_{k=2}^{\infty} k^{j} a_{k} z^{k}(j=\cdots,-2,-1,0,1,2, \cdots) .
$$

With the above operator $D^{j} f$, we introduce the subclass $S_{n}(\alpha)$.

Definition 3. The subclass $S_{n}(\alpha)$ of A consists of functions $f$, which satisfy

$$
\operatorname{Re}\left(\frac{D^{n+1} f(z)}{D^{n} f(z)}\right)>\alpha \quad(n=\cdots,-2,-1,0,1,2, \cdots)
$$

for $z \in \mathbb{U}$, where $0 \leq \alpha<1$.

Remark 1. Since $D^{0} f(z)=f(z), D^{1} f(z)=z f^{\prime}(z)$ and $D^{2} f(z)=z f^{\prime}(z)+z^{2} f^{\prime \prime}(z), f \in$ $S_{0}(\alpha)$ satisfies

$$
\operatorname{Re}\left(\frac{z f^{\prime}(z)}{f(z)}\right)>\alpha \quad(z \in \mathbb{U}),
$$


and $f \in S_{1}(\alpha)$ satisfies

$$
\operatorname{Re}\left(1+\frac{z f^{\prime \prime}(z)}{f^{\prime}(z)}\right)>\alpha \quad(z \in \mathbb{U}) .
$$

Therefore, $f \in S_{0}(\alpha)=S^{*}(\alpha)$ is starlike of order $\alpha$ in $\mathbb{U}$, and $f \in S_{1}(\alpha)=K(\alpha)$ is convex of order $\alpha$ in $\mathbb{U}$ (cf. Robertson [11]). Since $D^{-1} f$ is Alexander integral operator, $D^{-n} f(n=1,2,3, \cdots)$ is the generalization for Alexander integral operator (cf. Alexander [1]).

For a function $f \in A$, we introduce

$$
M_{p}(r, f)=\left\{\begin{array}{lc}
\left(\frac{1}{2 \pi} \int_{0}^{2 \pi}\left|f\left(r e^{i \theta}\right)\right|^{p} d \theta\right)^{\frac{1}{p}}, & (0<p<\infty) \\
\max _{|z| \leq r}|f(z)|, & (p=\infty) .
\end{array}\right.
$$

For the above $M_{p}(r, f)$, we define

$$
\mathcal{H}^{p}=\left\{f \in A:\|f\|_{p}=\lim _{r \rightarrow 1^{-}} M_{p}(r, f)<\infty\right\} .
$$

To discuss our problems, we have to introduce the following lemmas.

Lemma 1 (Wilken and Feng [12]). If $f \in S_{1}(\alpha)$, then $f \in S_{0}(\beta)$, where

$$
\beta=\beta(\alpha)= \begin{cases}\frac{2 \alpha-1}{2\left(1-2^{1-2 \alpha}\right)} \quad,\left(\alpha \neq \frac{1}{2}\right) \\ \frac{1}{2 \log 2}=0.7213 \ldots & ,\left(\alpha=\frac{1}{2}\right) .\end{cases}
$$

The result is sharp.

Lemma 2 (Eenigenburg and Keogh [13]). If $f \in S_{0}(\alpha)$ and

$$
f(z) \neq \frac{z}{\left(1-z e^{i \theta}\right)^{2}}
$$

then there exists $\delta=\delta(f)>0$ such that $\frac{f(z)}{z} \in \mathcal{H}^{\delta+\frac{1}{2(1-\alpha)}}$.

Lemma 3 (Nunokawa [14]). Let a function $p$ be analytic in $\mathbb{U}$ with $p(0)=1$. If $p$ satisfies

$$
\operatorname{Re}\left(p(z)+z p^{\prime}(z)\right)>\frac{1-2 \log 2}{2(1-\log 2)}=-0.629 \ldots \quad(z \in \mathbb{U})
$$

then $\operatorname{Rep}(z)>0(z \in \mathbb{U})$.

Lemma 4 (Duren [15]). If a function $p$ is analytic in $\mathbb{U}$ and $\operatorname{Rep}(z)>0(z \in \mathbb{U})$, then $p \in \mathcal{H}^{p}$ $(0<p<1)$.

Lemma 5 (Kim, Lee and Srivastava [16]). If $f \in A$ satisfies $z^{\gamma} f(z) \in \mathcal{H}^{p}(0<p<\infty)$ for some real $\gamma$, then $f \in \mathcal{H}^{p}(0<p<\infty)$.

Lemma 6 (Duren [15]). If $f \in A$ satisfies $f^{\prime} \in \mathcal{H}^{p}(0<p<1)$, then $f \in \mathcal{H}^{\frac{p}{1-p}}$.

Discussing our problems for Sălăgean operator, we need to introduce the following lemma due to Miller and Mocanu [17,18] (also, by Jack [19]).

Lemma 7 (Miller and Mocanu $[17,18])$. Let the function w given by

$$
w(z)=b_{n} z^{n}+b_{n+1} z^{n+1}+b_{n+2} z^{n+2}+\ldots, n \in \mathbb{N}
$$


be analytic in $\mathbb{U}$ with $w(0)=0$. If $|w(z)|$ attains its maximum value on the circle $|z|=r$ at a point $z_{0} \in \mathbb{U}$, then a real number $k \geq n$ exists, such that

$$
\frac{z_{0} w^{\prime}\left(z_{0}\right)}{w\left(z_{0}\right)}=k
$$

and

$$
\operatorname{Re}\left(1+\frac{z_{0} w^{\prime \prime}\left(z_{0}\right)}{w^{\prime}\left(z_{0}\right)}\right) \geq k
$$

The original results obtained by the authors and presented in this paper are contained in the next section. A new operator is introduced with Sălăgean differential operator as the inspiration. Using this newly introduced operator, a new class of functions denoted by $S_{n}(\alpha)$ is defined, with known classes as particular cases. Certain properties involving the applications of Sălăgean differential operator related to class $S_{n}(\alpha)$ are discussed in the theorems and corollaries. Examples are also included to prove the applications of the proved results.

\section{Main Results}

Now, we derive the following result.

Theorem 1. If $f \in S_{n}(\alpha)$, then $f \in S_{n-j}\left(\alpha_{j}\right)$, where $n>j \geq 0$ and

$$
\alpha_{j}= \begin{cases}\frac{2 \alpha_{j-1}-1}{2\left(1-2^{1-2 \alpha_{j-1}}\right)} & ,\left(\alpha_{j} \neq \frac{1}{2}\right) \\ \frac{1}{2 \log 2}=0.7213 \ldots & ,\left(\alpha_{j}=\frac{1}{2}\right) .\end{cases}
$$

Further, if

$$
D^{n-j} f(z) \neq \frac{z}{\left(1-z e^{i \theta}\right)^{2\left(1-\alpha_{j}\right)}}
$$

then there exists $\delta>0$, such that $D^{n-j} f \in \mathcal{H}^{\delta+\frac{1}{2\left(1-\alpha_{j}\right)}}$.

Proof. We note that if $f \in S_{n}(\alpha)$, then

$$
\operatorname{Re}\left(\frac{D^{n+1} f(z)}{D^{n} f(z)}\right)>\alpha_{0} \quad(z \in \mathbb{U}),
$$

where $\alpha_{0}=\alpha$. Since

$$
D^{n+1} f(z)=z\left(D^{n} f(z)\right)^{\prime}=z\left(D^{n-1} f(z)\right)^{\prime}+z^{2}\left(D^{n-1} f(z)\right)^{\prime \prime}
$$

and

$$
D^{n} f(z)=z\left(D^{n-1} f(z)\right)^{\prime},
$$

we see that

$$
\operatorname{Re}\left(\frac{D^{n+1} f(z)}{D^{n} f(z)}\right)=\operatorname{Re}\left(1+\frac{z\left(D^{n-1} f(z)\right)^{\prime \prime}}{\left(D^{n-1} f(z)\right)^{\prime}}\right)>\alpha_{0} \quad(z \in \mathbb{U}) .
$$


Applying Lemma 1, we say that

$$
\begin{aligned}
f \in S_{n}\left(\alpha_{0}\right) & \Leftrightarrow D^{n-1} f \in S_{1}\left(\alpha_{0}\right) \\
& \Rightarrow D^{n-1} f \in S_{0}\left(\alpha_{1}\right) \\
& \Leftrightarrow D^{n-2} f \in S_{1}\left(\alpha_{1}\right) \\
& \Rightarrow D^{n-2} f \in S_{0}\left(\alpha_{2}\right) \\
& \Leftrightarrow D^{n-3} f \in S_{1}\left(\alpha_{2}\right) \\
& \vdots \\
& \Leftrightarrow D^{n-j} f \in S_{0}\left(\alpha_{j-1}\right) \\
& \Rightarrow D^{n-j} f \in S_{1}\left(\alpha_{j}\right) .
\end{aligned}
$$

This implies that

$$
\operatorname{Re}\left(\frac{z\left(D^{n-j} f(z)\right)^{\prime}}{D^{n-j} f(z)}\right)=\operatorname{Re}\left(\frac{D^{n-j+1} f(z)}{D^{n-j} f(z)}\right)>\alpha_{j} \quad(z \in \mathbb{U}),
$$

that is, that $f \in S_{n-j}\left(\alpha_{j}\right)$. Further, applying Lemma 2, we see that if

$$
D^{n-j} f(z) \neq \frac{z}{\left(1-z e^{i \theta}\right)^{2\left(1-\alpha_{j}\right)}}
$$

then there exists $\delta>0$, such that $D^{n-j} f \in \mathcal{H}^{\delta+\frac{1}{2\left(1-\alpha_{j}\right)}}$.

Example 1. Let us consider a function $f$ belonging to the class $S_{3}(\alpha)$. Then $f \in S_{2}\left(\alpha_{1}\right)$ with (19), where

$$
\alpha_{1}= \begin{cases}\frac{2 \alpha-1}{2\left(1-2^{1-2 \alpha}\right)} & ,\left(\alpha \neq \frac{1}{2}\right) \\ \frac{1}{2 \log 2}=0.7213 \ldots & ,\left(\alpha=\frac{1}{2}\right) .\end{cases}
$$

Further, $f \in S_{1}\left(\alpha_{2}\right)$, where

$$
\alpha_{2}= \begin{cases}\frac{2 \alpha_{1}-1}{2\left(1-2^{1-2 \alpha_{1}}\right)} & ,\left(\alpha_{1} \neq \frac{1}{2}\right) \\ \frac{1}{2 \log 2}=0.7213 \ldots & ,\left(\alpha_{1}=\frac{1}{2}\right) .\end{cases}
$$

Also, $f \in S_{0}\left(\alpha_{3}\right)$, where

$$
\alpha_{3}= \begin{cases}\frac{2 \alpha_{2}-1}{2\left(1-2^{1-2 \alpha_{2}}\right)} & ,\left(\alpha_{2} \neq \frac{1}{2}\right) \\ \frac{1}{2 \log 2}=0.7213 \ldots & ,\left(\alpha_{2}=\frac{1}{2}\right) .\end{cases}
$$

If we consider the case of $\alpha=\frac{1}{4}$, then we have

$$
\begin{gathered}
\alpha_{1}=\frac{1}{4(\sqrt{2}-1)} \doteq 0.60355, \\
\alpha_{2}=\frac{3-2 \sqrt{2}}{4(\sqrt{2}-1)\left(1-2^{\frac{1-\sqrt{2}}{2}}\right)} \doteq 0.77436,
\end{gathered}
$$

and

$$
\alpha_{3} \doteq 0.8672
$$


Further, if we consider the case of $\alpha=\frac{1}{8}$, then

$$
\alpha_{1}=\frac{3}{8(\sqrt[4]{8}-1)} \doteq 0.55002
$$

and

$$
\alpha_{2}=\frac{7-4 \sqrt[4]{8}}{8(\sqrt[4]{8}-1)\left(1-2^{\frac{4 \sqrt[4]{8}-7}{4(\sqrt[4]{8}-1)}}\right)} \doteq 0.60607 .
$$

Remark 2. For some positive integer $j$, we know that

$$
\alpha_{j+1}=\frac{2 \alpha_{j}-1}{2\left(1-2^{1-2 \alpha_{j}}\right)},\left(\alpha_{j} \neq \frac{1}{2}\right)
$$

If we consider

$$
g\left(\alpha_{j}\right)=\alpha_{j+1}-\alpha_{j}=\frac{2 \alpha_{j}-1}{2\left(1-2^{1-2 \alpha_{j}}\right)}-\alpha_{j},\left(\alpha_{j} \neq \frac{1}{2}\right),
$$

$g(0)=\frac{1}{2}$ and $g(1)=0$. From this fact, we know that $\alpha_{j}<\alpha_{j+1}$ for $0 \leq \alpha_{j}<1$. This implies that

$$
0 \leq \alpha<\alpha_{1}<\alpha_{2}<\cdots<\alpha_{j}<\cdots<1 .
$$

Letting $j=n$ in Theorem 1 , we see

Corollary 1. If $f \in S_{j}(\alpha)$, then $f \in S_{0}\left(\alpha_{j}\right)$. If

$$
f(z) \neq \frac{z}{\left(1-z e^{i \theta}\right)^{2\left(1-\alpha_{j}\right)}},
$$

then there exists $\delta>0$, such that $f \in \mathcal{H}^{\delta+\frac{1}{2\left(1-\alpha_{j}\right)}}$.

\section{Next we have}

Theorem 2. If $f \in A$ satisfies

$$
\operatorname{Re}\left(\frac{D^{n+1} f(z)}{z}\right)=\frac{1-2 \log 2}{2(1-\log 2)}=-0.629 \ldots \quad(z \in \mathbb{U})
$$

for some $n \in \mathbb{N}$, then there exists $p_{j}$, such that $D^{n-j+1} f \in \mathcal{H}^{p_{j}}$, where

$$
p_{j}>\frac{1}{j-k+1} \quad(k=1,2,3, \cdots, j)
$$

and $j \leq n+1$.

Proof. If we define $p$ by

$$
p(z)=\frac{D^{n} f(z)}{z}
$$

then $p$ is analytic in $\mathbb{U}$ with $p(0)=1$. Since

$$
p(z)+z p^{\prime}(z)=\frac{D^{n+1} f(z)}{z},
$$

we see that

$$
\operatorname{Re}\left(\frac{D^{n+1} f(z)}{z}\right)=\operatorname{Re}\left(p(z)+z p^{\prime}(z)\right)>\frac{1-2 \log 2}{2(1-\log 2)} \quad(z \in \mathbb{U}) .
$$


Applying Lemma 3, we have that

$$
\operatorname{Re} p(z)=\operatorname{Re}\left(\frac{D^{n} f(z)}{z}\right)>0 \quad(z \in \mathbb{U}) .
$$

Using Lemma 4, we know that

$$
\frac{D^{n} f(z)}{z} \in \mathcal{H}^{p_{1}} \quad\left(0<p_{1}<\frac{1}{j}\right),
$$

that is, that $\left(D^{n-1} f(z)\right)^{\prime} \in \mathcal{H}^{p_{1}}$. By Lemma 6, we have that

$$
D^{n-1} f \in \mathcal{H}^{p_{2}} \quad\left(0<p_{2}=\frac{p_{1}}{1-p_{1}}<\frac{1}{j-1}\right) .
$$

Noting that

$$
D^{n-1} f(z)=z\left(D^{n-2} f(z)\right)^{\prime},
$$

we obtain that

$$
D^{n-2} f \in \mathcal{H}^{p_{3}} \quad\left(0<p_{3}=\frac{p_{2}}{1-p_{2}}<\frac{1}{j-2}\right) .
$$

Repeating the above, we have that

$$
D^{n-j+2} f \in \mathcal{H}^{p_{j-1}} \quad\left(0<p_{j-1}<\frac{1}{2}\right) .
$$

Finally, we get

$$
D^{n-j+1} f \in \mathcal{H}^{p_{j}} \quad\left(0<p_{j}<1\right) .
$$

Making $j=n+1$ in Theorem 2, we have

Corollary 2. If $f \in A$ satisfies

$$
\operatorname{Re}\left(\frac{D^{n+1} f(z)}{z}\right)>\frac{1-2 \log 2}{2(1-\log 2)}=-0.629 \ldots \quad(z \in \mathbb{U}),
$$

then, there exists $p_{n+1}$ such that $f \in \mathcal{H}^{p_{n+1}}\left(0<p_{n+1}<1\right)$.

Next, we derive

Theorem 3. If $f \in A$ satisfies

$$
\left|\frac{D^{n+2} f(z)}{D^{n+1} f(z)}-1\right|<\frac{5 \alpha-2 \alpha^{2}-1}{2 \alpha} \quad(z \in \mathbb{U}),(n \in \mathbb{N})
$$

for some real $\alpha\left(\frac{1}{3} \leq \alpha \leq \frac{1}{2}\right)$, or

$$
\left|\frac{D^{n+2} f(z)}{D^{n+1} f(z)}-1\right|<\frac{\alpha-2 \alpha^{2}+1}{2 \alpha} \quad(z \in \mathbb{U}),(n \in \mathbb{N})
$$

for some real $\alpha\left(\frac{1}{2} \leq \alpha<1\right)$, then $D^{n} f \in S_{0}(\alpha)$, that is, $D^{n} f$ is starlike of order $\alpha$ in $\mathbb{U}$. Further, if

$$
D^{n-j} f(z) \neq \frac{z}{\left(1-z e^{i \theta}\right)^{2\left(1-\alpha_{j}\right)}}
$$


then, there exists $\delta>0$ such that $D^{n-j} f \in \mathcal{H}^{\delta+\frac{1}{2\left(1-\alpha_{j}\right)}}$, where

$$
\alpha_{j}= \begin{cases}\frac{2 \alpha_{j-1}-1}{2\left(1-2^{1-2 \alpha_{j-1}}\right)} & ,\left(\alpha_{j-1} \neq \frac{1}{2}\right) \\ \frac{1}{2 \log _{2}}=0.7213 \ldots & ,\left(\alpha_{j-1}=\frac{1}{2}\right)\end{cases}
$$

and $j \leq n$.

Proof. Define a function $w$ by

$$
\frac{D^{n+1} f(z)}{D^{n} f(z)}=\frac{1+(1-2 \alpha) w(z)}{1-w(z)} \quad(w(z) \neq 1) .
$$

It follows from the above that

$$
\frac{D^{n+2} f(z)}{D^{n+1} f(z)}-\frac{D^{n+1} f(z)}{D^{n} f(z)}=\frac{(1-2 \alpha) z w^{\prime}(z)}{1+(1-2 \alpha) w(z)}+\frac{z w^{\prime}(z)}{1-w(z)} .
$$

Therefore, we have that

$$
\frac{D^{n+2} f(z)}{D^{n+1} f(z)}-1=\left(\frac{w(z)}{1-w(z)}\right)\left\{2(1-\alpha)+\frac{z w^{\prime}(z)}{w(z)}\left(1+\frac{(1-2 \alpha)(1-w(z))}{1+(1-2 \alpha) w(z)}\right)\right\} .
$$

Suppose that there exists a point $z_{0} \in \mathbb{U}$, such that

$$
\max _{|z| \leq \mid z_{0}}|w(z)|=\left|w\left(z_{0}\right)\right|=1 \quad\left(w\left(z_{0}\right) \neq 1\right) .
$$

Then, Lemma 7 say that $w\left(z_{0}\right)=e^{i \theta}$ and $z_{0} w^{\prime}\left(z_{0}\right)=k w\left(z_{0}\right)(k \geq 1)$. This implies that

$$
\begin{aligned}
\left|\frac{D^{n+2} f\left(z_{0}\right)}{D^{n+1} f\left(z_{0}\right)}-1\right| & =\left|\frac{e^{i \theta}}{1-e^{i \theta}}\right|\left|2(1-\alpha)+k\left(1+\frac{(1-2 \alpha)\left(1-e^{i \theta}\right)}{1+(1-2 \alpha) e^{i \theta}}\right)\right| \\
& \geq \frac{2\left(1-\alpha_{0}\right)+k}{\left|1-e^{i \theta}\right|}-\frac{k|1-2 \alpha|}{\left|1+(1-2 \alpha) e^{i \theta}\right|} \\
& \geq \frac{2\left(1-\alpha_{0}\right)+k}{2}-\frac{k|1-2 \alpha|}{2 \alpha} .
\end{aligned}
$$

If $\frac{1}{3} \leq \alpha<\frac{1}{2}$, then

$$
\left|\frac{D^{n+2} f\left(z_{0}\right)}{D^{n+1} f\left(z_{0}\right)}-1\right| \geq \frac{5 \alpha-2 \alpha^{2}-1}{2 \alpha}
$$

and if $\frac{1}{2} \leq \alpha<1$, then

$$
\left|\frac{D^{n+2} f\left(z_{0}\right)}{D^{n+1} f\left(z_{0}\right)}-1\right| \geq \frac{\alpha-2 \alpha^{2}+1}{2 \alpha} .
$$

This contradicts our condition of the theorem. Thus we say that $|w(z)|<1$ for all $z \in \mathbb{U}$. From the definition (57) for $w$, we obtain that

$$
\operatorname{Re}\left(\frac{D^{n+1} f(z)}{D^{n} f(z)}\right)>\alpha \quad(z \in \mathbb{U}) .
$$

This means that $D^{n} f \in S_{0}(\alpha)$. Letting $\alpha=\alpha_{0}$ and using Lemma 1 , we obtain $D^{n-j} f \in$ $S_{0}\left(\alpha_{j}\right)$, where $\alpha_{j}$ is given by (56). Applying Lemma 2, we know that if

$$
D^{n-j} f(z) \neq \frac{z}{\left(1-z e^{i \theta}\right)^{2\left(1-\alpha_{j}\right)}},
$$


then, there exists $\delta>0$ such that $D^{n-j} f \in \mathcal{H}^{\delta+\frac{1}{2\left(1-\alpha_{j}\right)}}$.

Making $j=n$ in Theorem 3, we have

Corollary 3. If $f \in A$ satisfies

$$
\left|\frac{D^{n+2} f(z)}{D^{n+1} f(z)}-1\right|<\frac{5 \alpha-2 \alpha^{2}-1}{2 \alpha} \quad(z \in \mathbb{U}),
$$

for some real $\alpha\left(\frac{1}{3} \leq \alpha \leq \frac{1}{2}\right)$, or

$$
\left|\frac{D^{n+2} f(z)}{D^{n+1} f(z)}-1\right|<\frac{\alpha-2 \alpha^{2}+1}{2 \alpha} \quad(z \in \mathbb{U}),
$$

for some real $\alpha\left(\frac{1}{2} \leq \alpha<1\right)$, then $D^{n} f \in S_{0}(\alpha)$. If

$$
f(z) \neq \frac{z}{\left(1-z e^{i \theta}\right)^{2\left(1-\alpha_{n}\right)}}
$$

then, there exists $\delta>0$, such that $f \in \mathcal{H}^{\delta+\frac{1}{2\left(1-\alpha_{n}\right)}}$.

\section{Conclusions}

Inspired by the classic and well-known Sălăgean differential operator, a new operator is introduced in Definition 2. By applying this operator, a new class of functions is defined, denoted by $S_{n}(\alpha)$. It is shown that classes of starlike and convex functions of the order $\alpha$ are obtained for specific values of $n$. Some interesting problems concerning the class $S_{n}(\alpha)$ are discussed in the theorems and corollaries. One example is given as an application for special cases of $n$ for the class $S_{n}(\alpha)$. The new operator defined in this paper can be used to introduce other certain subclasses of analytic functions. Quantum calculus can be also associated for future studies, as can be seen in paper [20] regarding the Sălăgean differential operator and involving symmetric Sălăgean differential operator in paper [21]. Symmetry properties can be investigated for this operator, taking the symmetric Sălăgean derivative investigated in [22] as inspiration.

Author Contributions: Conceptualization, S.O., H.Ö.G. and G.I.O.; Investigation, S.O., H.Ö.G. and G.I.O.; Methodology, S.O.; Writing—original draft, S.O.; Writing—review and editing, H.Ö.G. and G.I.O. All authors have read and agreed to the published version of the manuscript.

Funding: This research received no external funding.

Institutional Review Board Statement: Not applicable.

Informed Consent Statement: Not applicable.

Data Availability Statement: Not applicable.

Conflicts of Interest: The authors declare no conflict of interest.

\section{References}

1. Alexander, J.W. Functions which map the interior of the unit circle upon simple regions. Ann. Math. 1915, 17, 12-22. [CrossRef]

2. Bernardi, S.D. Convex and starlike univalent functions. Trans. Am. Math. Soc. 1969, 135, 429-446. [CrossRef]

3. Libera, R.J. Some classes of regular univalent functions. Proc. Am. Math. Soc. 1965, 16, 755-758. [CrossRef]

4. Acu, M.; Oros, G. Starlikeness condition for a new differential-integral operator. Mathematics 2020, 8, 694. [CrossRef]

5. Oros, G.I.; Alb Lupas, A. Sufficient conditions for univalence obtained by using Briot-Bouquet differential subordination. Math. Stat. 2020, 8, 26-136. [CrossRef]

6. Páll-Szabó, Á.O.; Wanas, A.K. Coefficient estimates for some new classes of bi-Bazilevič functions of Ma-Minda type involving the Sălăgean integro-differential operator. Quaest. Math. 2021, 44, 495-502.

7. Alb Lupaş, A.; Oros, G.I. Strong differential superordination results involving extended Sălăgean and Ruscheweyh Operators. Mathematics 2021 9, 2487. [CrossRef] 
8. Alb Lupaş, A.; Oros, G.I. On Special Differential Subordinations Using Fractional Integral of Sălăgean and Ruscheweyh Operators. Symmetry 2021 13, 1553. [CrossRef]

9. Aouf, M.K.; Mostafa, A.O.; Madian, S.M. Fekete-Szegö properties for quasi-subordination class of complex order defined by Sălăgean operator. Afr. Mat. 2020, 31, 483-492. [CrossRef]

10. Sălăgean, G.S. Subclasses of Univalent Functions. In Complex Analysis_Fifth Romanian-Finnish Seminar, Part I Bucharest; Lecture Notes in Mathematics 1013; Springer: Berlin/Heidelberg, Germany, 1981; pp. 362-372.

11. Robertson, M.I. On the theory of univalent functions. Ann. Math. 1936, 37, 374-408. [CrossRef]

12. Wilken, D.R.; Feng, J. A remark on convex and starlike functions. J. Lond. Math. Soc. 1980, 2, 287-290. [CrossRef]

13. Eenigenburg, P.J.; Keogh, F.R. The Hardy class of some univalent functions and their derivatives. Mich. Math. J. 1970, 17, 335-346. [CrossRef]

14. Nunokawa, M. On starlikeness of Libera transformation. Complex Var. Elliptic Equs. 1991, 17, 79-83. [CrossRef]

15. Duren P.L. Univalent Functions; Grundlehren der Mathematischen Wissenschaften, Band 259; Springer: New York, NY, USA; Berlin/Heidelberg, Germany; Tokyo, Japan, 1983.

16. Kim, Y.C.; Lee, K.S.; Srivastava, H.M. Certain classes of integral operators associated with the Hardy space of analytic functions. Complex Var. Elliptic Equs. 1992, 20, 1-12. [CrossRef]

17. Miller, S.S.; Mocanu, P.T. Second order differential inequalities in the complex plane. J. Math. Anal. Appl. 1978, 65, 289-305. [CrossRef]

18. Miller, S.S.; Mocanu, P.T. Differential Subordinations. In Theory and Applications; Marcel Dekker Inc.: New York, NY, USA, 2000.

19. Jack, I.S. Functions starlike and convex of order $\alpha$. J. Lond. Math. Soc. 1971, 2 , 469-474. [CrossRef]

20. Govindaraj, M.; Sivasubramanian, S. On a class of analytic functions related to conic domains involving q-calculus. Anal. Math. 2017, 43, 475-487. [CrossRef]

21. Ibrahim, R.W.; Elobaid, R.M.; Obaiys, S.J. Geometric Inequalities via a Symmetric Differential Operator Defined by Quantum Calculus in the Open Unit Disk. J. Funct. Spaces 2020, 2020, 6932739. [CrossRef]

22. Ibrahim, R.W.; Darus, M. Univalent functions formulated by the Sălăgean-difference operator. Int. J. Anal. Appl. 2019, 17, 652-658. 